\title{
A computer-assisted bacteriology reporting and information system
}

\author{
D. A. MITCHISON, J. H. DARRELL, AND RUTH MITCHISON \\ From the Department of Bacteriology, Royal Postgraduate Medical School, London, UK
}

SUMMARY A computer system for reporting and recording all specimens processed by the routine bacteriology laboratory at the Royal Postgraduate Medical School is described. Features of interest are the method of input using a mixture of 3-character alphanumeric codes and numbers, cumulative reporting to the wards, and selective listing of relevant previous results for the patient, which is available to technical and supervisory staff during processing of the specimen. The relative value to the wards and the laboratory of each type of information transfer has been assessed. Overall the use of a computer has resulted in higher quality bench work and more accurate reporting. It seems little more expensive than a previous manual system, although it has transferred work from the technical to the clerical staff.

A computer-assisted system for reporting bacteriological results to clinicians and for providing feedback to the laboratory has been developed at the Royal Postgraduate Medical School over the past five years. It has been running in its completed version for the past year, so that we are now in a position to assess its value. The computer used, an Elliott 4120, is obsolete, and the problems encountered in having to rely solely on magnetic tape for bulk storage of records are irrelevant to modern computer technology. Nevertheless the system as seen from the user's standpoint is sophisticated and has taught us much about the relative value of different types of information transferral within the laboratory and between the laboratory and the wards. There are several problem areas worth considering, some of which are common to all medical laboratory information systems and others which are specific to microbiology systems. This report will be concerned mainly with these questions of system application.

\section{The system}

Several important decisions about the system were taken early in its development. Firstly, we wished to make cumulative reports available to our clinical colleagues, which meant that the report format had to be compact, simple, and similar for the different types of specimen and that particular emphasis had to be placed on maintaining complete patient records.

Received for publication 14 December 1977
Secondly, the amount of information per record that we could store was limited, as was the supervisory skill necessary to ensure reliability and computer readability of the input. We therefore decided to record only those items that were to be reported (except for some additional sensitivity test results) and not information such as the details of sugar reactions and other identification tests of interest only within the laboratory. Thirdly, the computer had to be batch operated with a low cost input system based on paper tape produced by teletypes. Fourthly, we considered that transfer of information within the laboratory was potentially as important as reporting to the wards.

Specimens totalling about 60000 per year are received in the laboratory accompanied by a form which gives details of identification of patients, including a hospital (case) number in most but not all instances (Fig. 1). The form is used as a work sheet in the laboratory and for recording the eventual coded report. On arrival, a unique check lettered laboratory number, computer printed on six replicate self-adhesive labels, is affixed to the specimen, the form, and plates of culture medium used. The specimen is also allocated by technical staff to one of 19 types (Table 1). The laboratory number, specimen type, hospital number, and other identification details are then punched on to paper tape by clerical staff. From this information is produced:

1 A day book. This lists specimen date, hospital number, sex, age, ward, consultant, specimen type, laboratory number, surname and forename(s), arranged in alphabetical patient name order. 


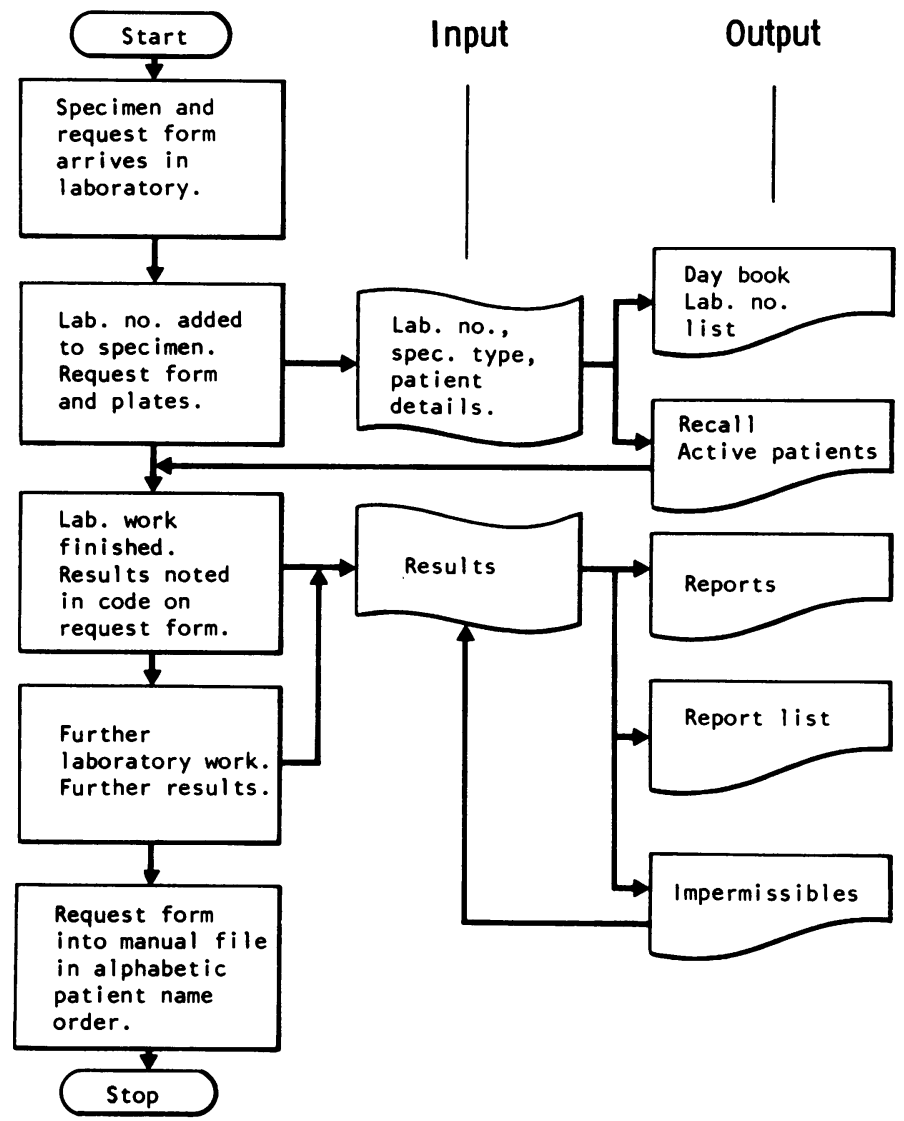

Fig. 1 Simplified flow chart of system

2 A laboratory number list. This contains the same information as the day book arranged by order of the laboratory number and is used by technicians wishing to identify plates separated from their forms and to identify the initial omission of occasional specimens from the system.

3 A list of all specimens received from any of a group of up to 20 'active' patients (or wards) who are of special interest to supervisory staff.

4 Recall. The results of previous bacteriology are printed for certain types of specimen. Currently these comprise all types of specimen previously examined from patients with new blood cultures and antibiotic assays. In addition, all urines from patients who have previously had at least one urine with a significant growth of organisms cause recall of previous results of urines only, and similarly all peritoneal dialysis fluids selectively recall previous results on peritoneal dialysis fluids only. A similar indication of a previous positive result in any venereal serology test is given on receipt of a further serology request for the patient.

When laboratory work has been completed sufficiently for a report to be sent, the results are written on the request form and these are punched on to paper tape by clerical staff (Fig. 2). The entries consist either of numbers, sometimes followed by a letter, or of 'code' entries. A code entry is a mnemonic group of up to three alphanumeric characters starting with a letter, such as SAU for Staphylococcus aureus, GEN for gentamicin, or CFT for complement fixation test. This entry is matched into an index containing all valid codes, translated into a number held in the computer record and output, if required, as a string of characters often much longer than the input code. There are nine independent indexes with- 
Table 1 Types of specimen

\begin{tabular}{ll}
\hline Code & Type of specimen \\
\hline STE & Sterility test \\
TS & Throat, nose, or mouth swab \\
GS & Gynaecological swab \\
F & Faeces \\
U & Urine \\
PAR & Parasitology \\
MYC & Mycology \\
TBS* & Sputum for mycobacteria \\
TBP* & Pus or fluid for mycobacteria \\
TBU* & Urine for mycobacteria \\
TBM* & Tissue or miscellaneous for mycobacteria \\
WR & Serological test for syphilis \\
SE & Other serological test \\
ANT & Antibiotic assay \\
SP & Sputum \\
C & Cerebrospinal fluid \\
B & Blood culture \\
P & Pus, tissue, fluid, etc \\
PDF & Peritoneal dialysis fluid \\
\hline
\end{tabular}

*All output on reports as TB.

in the system which hold, among other items, the names of consultants, wards, and antibiotics as well as bacterial species, types of test, and anatomical sites. These codes simulate and replace the notes normally recorded by technicians, are economical to produce, easy for junior technical staff to learn, and less error prone than numerical entries. The format of the report is similar for all types of specimen. It starts with a 'special code' entry, which gives the type of specimen or the result of a microscopic examination followed by a 'special number' giving, for instance, the amount of pus or acid-fast bacilli or white cells in a cerebrospinal fluid. The special code and number can be followed by up to seven pairs of entries consisting of a code for 'organisms', which also includes names of serological tests and anatomical sites followed by a 'profusion' number, which may give profusion of growth, the result of a serological test, or an antibiotic concentration. Any of these pairs of 'organisms' and 'profusion' entries may be followed by sensitivity test results. Finally, the record can end with a comment section of free text. About $45 \%$ of the reports are specified by a single standard entry code from 24 available (coded as S1-S24), each of which causes a group of entries, usually for negative results, to be inserted into the record. All entries are terminated with a semicolon followed by run outs on the paper tape. Inclusion of ' $/$ ' after the semicolon deletes the record, making error correction as simple as it is with punch cards.

Reports are printed in twice-daily runs (Fig. 3). Individual reports contain all results from the patient entered in the run concerned. At four days after the first specimen, and thereafter at weekly intervals (provided reports are still being issued), a cumulative report is produced containing current results as well as those for all tuberculosis and blood culture specimens for the past six to seven months and for all other specimens for the past two to three months. A report list to assist the laboratory in answering telephone enquiries is printed containing all current reports arranged by alphabetic patient name order. A list of impermissible results is also produced by the occurrence of specific combinations of bacterial species with an inconsistent antibiotic sensitivity test result, such as Streptococcus pyogenes reported as resistant to penicillin. During the course of a twicedaily run, records may be edited, deleted, or merged. New entries can be added to any index, and the list of active patients or wards may be modified. After reports have been printed, the request forms are filed manually in separate dockets for each patient in alphabetic name order, so that records can be rapidly accessed for reviews on any patient.

The computer records are held on magnetic tape, in order first of hospital number and then of laboratory number. The current tapes are archived monthly, specimens over 2 months old (6 months for blood culture and examination for mycobacteria) being removed, if complete, to storage tapes, which hold all records for a six-month period. The basic record for a patient remains until it has been dormant for nine months (five years if a positive venereal serology result has been entered). Lists of incomplete results are printed. Routine statistics on the number and types of specimen are produced. The report lists are stored, and an index to them is eventually prepared from the storage tape so that manual as well as computer access is available to archives. At weekly intervals lists are printed of positive blood cultures for the past month and of spacimens from fee-paying patients for the past week.

\section{Problem areas}

\section{CUMULATIVE REPORTING}

Cumulative reports are issued for all specimens other than those for quality control and from general practitioners. Cumulative reporting demands effective systems, firstly to ensure that a new specimen record is attached to the file of the correct patient, and secondly to detect and merge records with different hospital numbers for the same patient. Maintenance of accurate patient files is aided by the use of addressograph labels, with identification details, on the majority of request forms. When new records are merged by the computer, they are compared with identification details for the same hospital number in the old file. Warning messages are output if there is any difference in the spelling of surname or forename, in sex or in age, but the new record is 


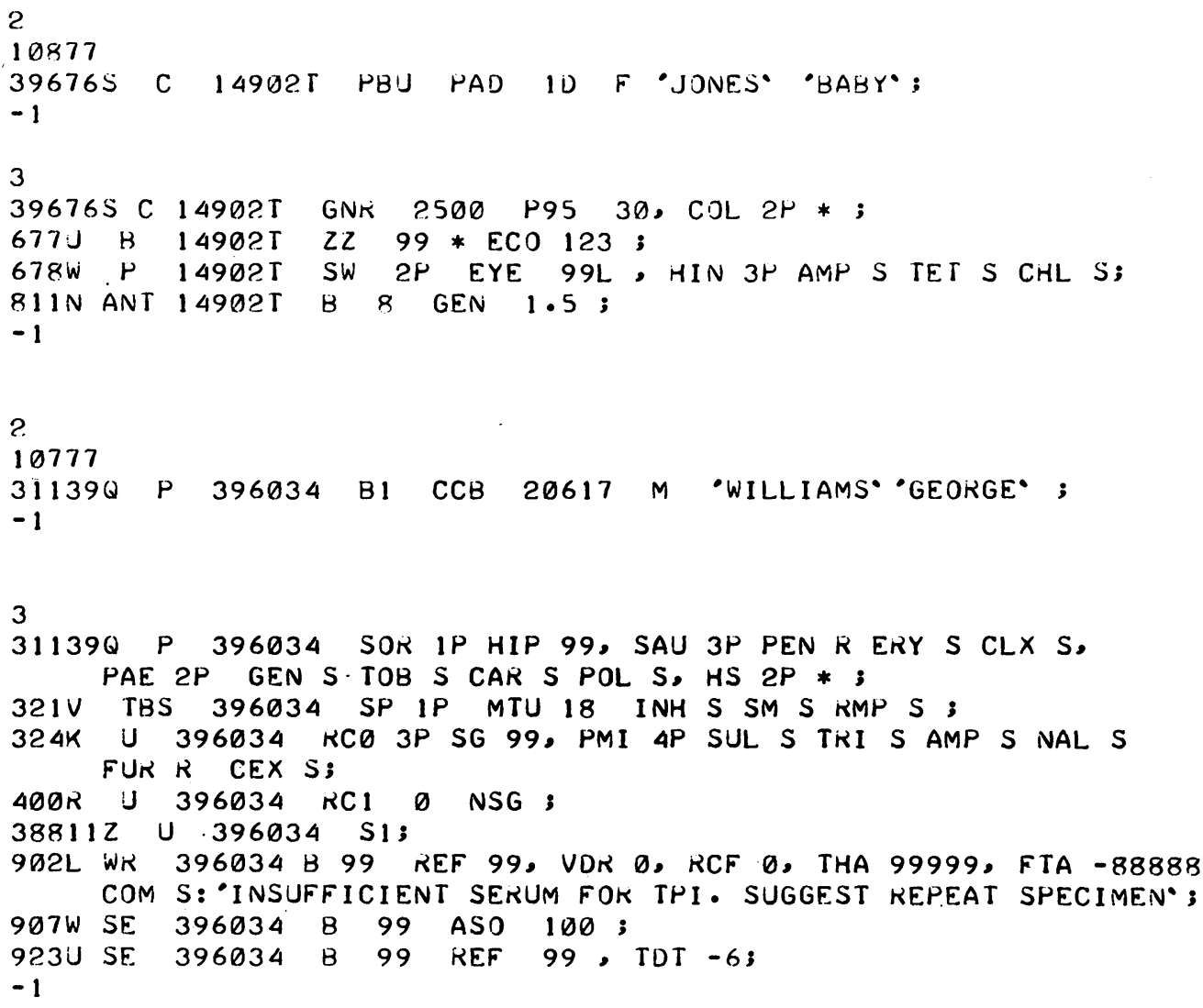

Fig. 2 Teletype entries for reports shown in Fig. 3.

The first entry records the arrival (indicated by '2') of a specimen on 1 August 1977 from Baby Jones. The data listed are: laboratory no., specimen type, hospital no. (temporary), ward, consultant's initials, age, sex, name, forename. Similar entries would be made for all other specimens. There follow results entries (indicated by '3') for four specimens. The data listed include: laboratory no., specimen type, hospital no., special code, special no., organism, profusion. A comma allows entry of an additional organism and profusion. An asterisk generates FURTHER REPORT TO FOLLOW. ZZ and 99 are nul entries. Special features are:

39676 The first 'organism' entry P95 generates WBC 95\% POLYS RBC.

39677 The numbers of the blood culture bottles $(1,2$, and 3$)$ yielding $E$. coli are entered in 'profusion' as 123.

39678 For several specimen types, PUS is automatically printed before the 'special no.' entry. 99L in 'profusion' results in LEFT.

39811 For antibiotic assays, the interval between the last dose and taking blood (8.0) is in 'special no.' and the concentration (1.5) in 'profusion'.

A single specimen arrival entry for George Williams is followed by the results entries for eight specimens. Special features are:

31321 For TB specimens, AFB precedes the 'special no.' entry.

31811 The 'special code' entry S1 results in a standard no pus, no growth output for urines.

31902 The dummy sensitivity test entry COM S and the colon are necessary for insertion of the free comment.

31907 Automatic printing of UNITS or TITRE is specified by the serological test being 31923 reported.

All entries are from fictitious patients. 


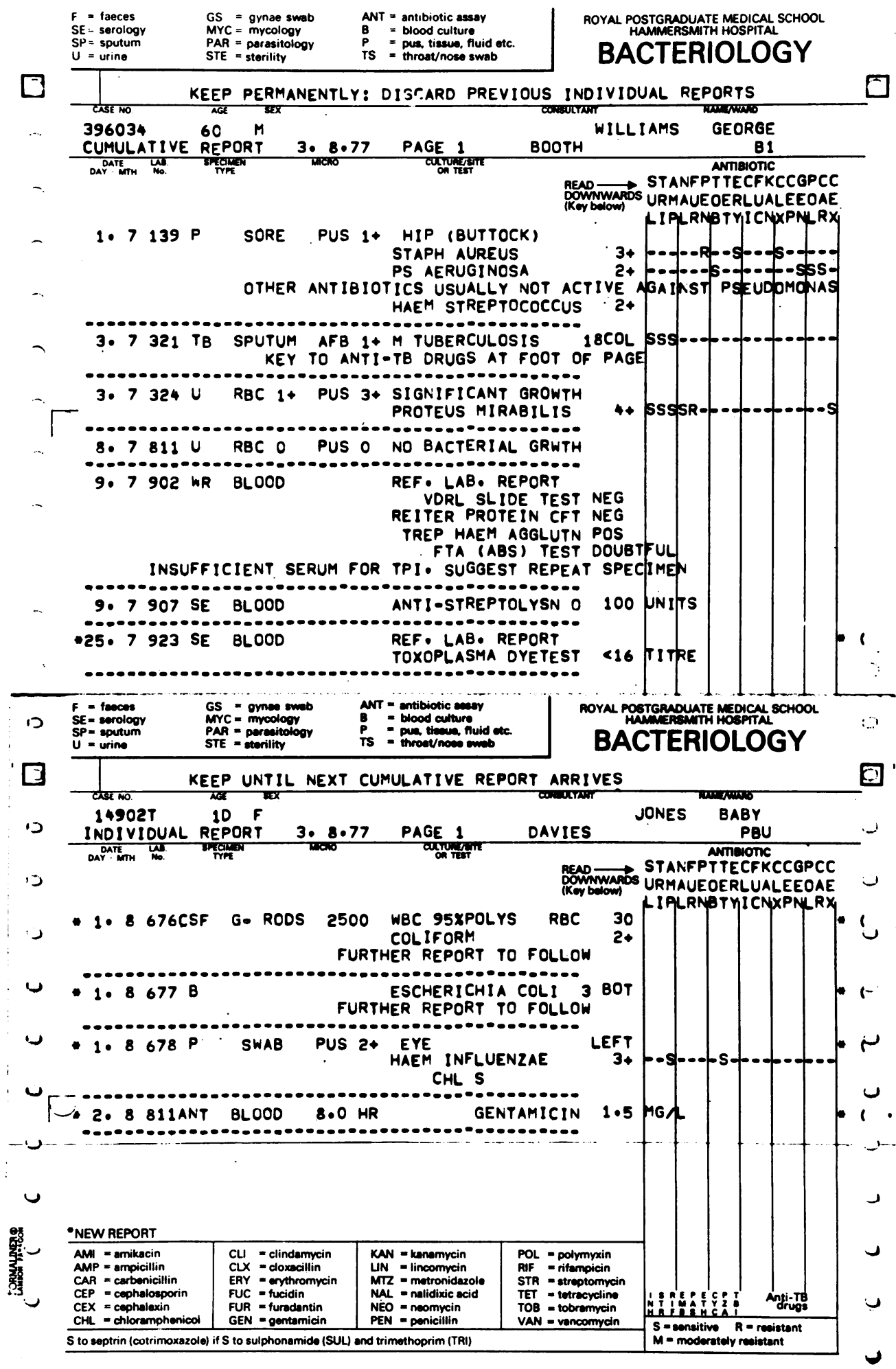

Fig. 3 A cumulative and an individual report.

In the original, the black line printer output is on forms pre-printed in light orange. An unused section of the lower report, above the key antibiotics, has been cut out. 
rejected only if it differs in the first three characters of the surname. A further check occurs at filing of the completed request form. In practice, it has been exceedingly rare for a specimen record to be filed in the record of a different patient, the only examples being in baby-mother pairs when a specimen from the baby has been sent in with the identification details of the mother. Multiple records for the same patient may occur because about $20 \%$ of request forms are received without a hospital number. After checking from a daily computer listing that a temporary number has not already been allocated to the patient, these specimens are given a temporary number from a manual register, and a card with the number and the patient's name is placed in the manual patient file. When a request form is eventually filed, its hospital number is checked against this card to detect the use of more than one temporary number or the issue of a permanent hospital number to the patient. A small proportion of patients are allocated two or more permanent numbers, and this again may be detected at filing. A computer program searches at weekly intervals and detects about six additional multiple records.

\section{ANATOMICAL SITE}

Swabs, fluids, pus, tissue biopsies, and miscellaneous objects are received from various anatomical sites in the body. Recording of this information on the request form is often incomplete and poorly legible so that a junior technician may not be able to enter the information correctly into the computer record. A check by a senior technician or medical graduate is essential. After considering various alternative methods of recording this information, a system has been evolved using up to three separate entries in the format spaces for special code, organisms, and profusion. The first entry specifies the type of material examined, for example, swab, pus, drain, catheter, fluid, aspirate. The second entry specifies the anatomical site from a list of about 60 alternatives. The third entry specifies left, right, top, mid, bottom, pre, or post if required. The free comment facility is available to supplement these entries. This system is most often used for the $P$ specimen type (Table 1) but also for TBP, TBM, MYC, and occasionally other types.

\section{CHECKING}

The initial coding of results on all specimens, together with the associated bacteriology, is always checked by a senior member of staff, usually a medical graduate. The entry is then punched, and processed through the computer system, including an initial validation run. A second check is made on the report list by clerical staff to see that sensitivity test results agree with those on the work sheet; errors? are very rare, occurring in about $0.2 \%$ of specimens 0 Finally, medical staff check the report lists and change these lists and the corresponding reports by hand, where necessary. From the report lists it care be estimated that a change was necessary in aboue $1.2 \%$ of reports in each run. The changes were usually made for bacteriological reasons, particularly the suppression of inappropriate sensitivity tese results, and rarely (about $0.3 \%$ ) from miscoding or mispunching, most often an incorrect use of the symbol indicating a further report to follow. Thछ corresponding corrections are made on the compute? record using a special editing entry.

\section{The value of the system}

Although accurate costing of the system is im possible, approximate estimates can be made of gains and losses on changing from the earlier arrangements, in which reports were prepared bye technicians partly with preprinted adhesive labels and partly in handwriting. On the credit side, allo technicians spend less time on writing reports; eachpo gains perhaps 30-60 minutes a day. On the debit sidep about half an additional clerical post is required, anç a small proportion of the time of an experience of programmer is necessary for systems maintenance To these debits must be added computing costs which would be lower on a modern machine. Or balance costs have remained similar or have in $\frac{3}{3}$ creased slightly. However, certain major advantages which cannot easily be costed, accrue:

1 The reports, including cumulative reports, are popular with clinical colleagues, particularly as they are brief and easily legible. A simple questionnaire was distributed through departmental secretaries with the request that it be given mainly to clinica@ registrars and senior registrars who would have remembered the older manual system and only to those consultants likely to have the actual manage $\rightarrow$ ment of their patient's notes. In the event, about hal of the expected forms were returned, dependento mainly on the persistence of the secretaries; they were completed by 33 clinicians, of whom nine weres consultants. Of the 27 with experience of the old system, 12 thought the computer system a consider able improvement and 12 an improvement, while tw thought it less valuable. Of the 31 who answered the question on cumulative reporting (assessment on $\mathrm{a}^{\mathrm{P}}$ five-point scale), 11 found it of considerable value 19 of value, and one of no value; none found it hindrance.

2 The reports are more accurate, due to the discipline engendered and to greater ease of finat checking. 
3 There is greater awareness within the laboratory of important current results and of previous findings, leading to more cost effective bench work and to a more useful dialogue with clinical colleagues.

4 Telephone enquiries are answered more rapidly and effectively.

5 Stored data are much easier to analyse. This has already proved of considerable value, both in estimating from what source the laboratory work load arises and also for research projects.

An approximate cost/benefit analysis of the various types of output from the system is set out in Table 2,

Table 2 Work load, value, and likely retention in on-line systems of different types of computer output

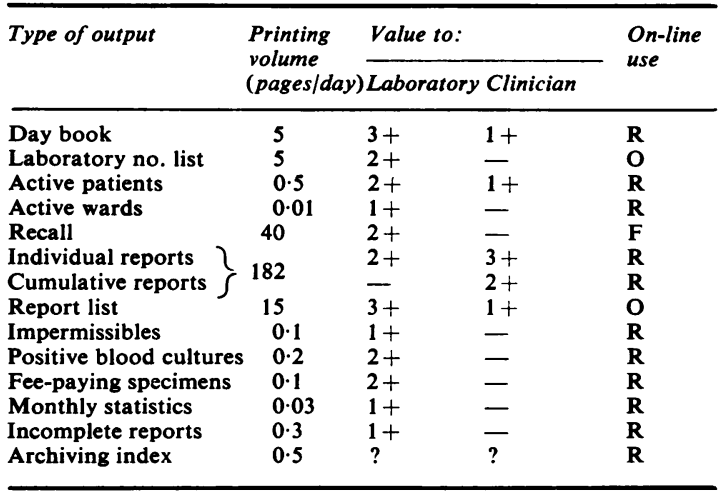

$\mathbf{R}=$ retain, $\mathbf{F}=$ some features to be retained, $\mathbf{O}=$ omit.

in which cost is estimated as printing volume and benefit on an arbitrary scale of value, with separate assessments for the laboratory and the clinicians. The printing of reports is much the largest form of output. It is of high $(3+)$ value to clinicians but is also of moderate value $(2+)$ to the laboratory because it saves the time of the technical staff. The day book and the report list are of high value in the laboratory, and they also save the time of clinicians making telephone enquiries. Other outputs of moderate value to the laboratory are the laboratory number list, the active patient list, and the weekly listings of previous positive blood cultures and specimens from fee-paying patients. It is too early to say how useful the archiving system will prove to be.

\section{Change to an on-line system}

The current system is batch operated so that rapid access to the file of a patient cannot be obtained. As a result a manual file containing work sheets (the backs of the request forms) is maintained in patient name order. There is also a considerable volume of computer listing to meet the needs for information that might be wanted rather than only information actually required. An on-line system would allow immediate access to a patient's computer file and would therefore render a manual file unnecessary. It would also reduce the amount of computer listing, as indicated in Table 2 . The laboratory number list could probably be omitted. Most of the recall would also be unnecessary, though it would seem desirable to retain the marking of a patient's record by the occurrence of a finding indicating a specific type of disease, such as a urinary tract infection or positive venereal serology. When the relevant type of specimen was received, a marked record would indicate the desirability of calling for a VDU display of previous records for the patient at the appropriate time, but without an actual list. Daily report lists would also be unnecessary, though an eventual hard copy would be desirable, perhaps at the time of archiving of the records. The advantages of an online system are considerable both in the speed with which it would provide access to patient's records and in the reduced volume of listing. New computer systems are likely to be on-line despite the technical problems involved. One of the merits of the current method for data entry, using a combination of mnemonic codes and numbers, is the ease with which it could be adapted to on-line use.

\section{Discussion}

There have been several recent reports of computer systems for reporting and storing results from microbiological laboratories (Andrews and Vickers, 1974; Vermeulen et al., 1974; Farrar et al., 1975; Kunz et al., 1975; Goodwin and Smith, 1976). The system we have described is unique in selectively recalling past results on the patient early on the day after receipt of a relevant specimen so that they are immediately available for guiding the bench work and later for interpreting the results. This recall arrangement resembles scheme $D$ of the Working Party of the Association of Clinical Pathologists (1968), though the latter scheme, based on experience in chemical pathology (Wootton, 1965), instigates recall at the time of reporting rather than at the time of processing the specimen. Even with an on-line system it seems important to us that the patient's record should be flagged to indicate the occurrence of previous positive pathology, such as a urinary tract infection, and should automatically indicate to laboratory staff the type of the previous disease and the need to call up previous records of the patient.

Our cumulative reporting system was popular among most of our clinical colleagues. However, only a few other microbiology systems have been described that provide cumulative reports to the 
wards and these were in America (Spraberry, 1969; Vermeulen et al., 1974; Kunz et el., 1975). We do not know whether it is preferable to arrange cumulative reports primarily in date order, as we have done, or to divide them first according to the type of specimen.

The method of input to any computer system is decisive in whether the system is economical, relatively error-free, and easily comprehensible to technical staff. Many systems have used a code entry either largely numerical (Alexander et al., 1970; Harvey et al., 1972; Farrar et al., 1975; Goodwin and Smith, 1976; Whitby and Blair, 1970) or, as in our system, partly with mnemonic alphanumeric codes and partly with numbers (Kunz et al., 1975; Gaya and Thirlwall, 1976). We believe that the mnemonic, self-checking nature of our coding is a considerable improvement on purely numerical methods. Other systems use optical mark sensing devices (Ayliffe and Chalke, 1973; Andrews and Vickers, 1974); these suffer from the disadvantages of high capital cost of equipment, liability to error (Gaya and Thirlwall, 1976), and relative inflexibility, since it is much more expensive and time-consuming to redesign mark sensing forms than to change the vocabularies contained in our coding indexes. We believe it is a great advantage to have trained clerical staff punching the codes generated by technicians since they detect and correct a high proportion of the initial coding errors. In our experience, few technicians are prepared to refer at frequent intervals to written instructions for coding and they make mistakes with unusual reports. The additional money spent on clerical staff has been more than saved by reducing the work load on our technical staff.

We are grateful to Miss Irene Allison, who contributed to the initial systems design and programming, and to Mrs Jenny Perry, who assisted in its later development.

\section{References}

Alexander, M. K., Connigale, J., Johnson, T., Poulter, I. R., and Wakefield, J. (1970). A data processing system for hospital bacteriology. Journal of Clinical Pathology, 23, 77-80.

Andrews, H. J., and Vickers, M. (1974). An assessment of one year of computer-assisted microbiology reporting at Charing Cross Hospital. Journal of Clinical Pathology, 27, 185-191.

Association of Clinical Pathologists (1968). Data processing in clinical pathology. Journal of Clinical Pathology, 21, 231-301.

Ayliffe, P. F., and Chalke, R. (1973). A computer system to perform record handling and reporting for a hospital microbiology laboratory. Medical Laboratory $\widetilde{\Phi}_{\overparen{Q}}$ Technology, 30, 363-371.

Farrar, J. L., Brogan, T. D., Moulding, T., Taylor, R., ڤ and Page, M. (1975). Use of a computer for producing $\vec{O}$ microbiological reports and for data storage and processing. Journal of Clinical Pathology, 28, 580-586. $\vec{\omega}$

Gaya, H., and Thirlwall, J. (1976). Data handling in clinical microbiology: problems and solutions. In 2nd International Symposium on Rapid Methods and Automation in Microbiology, Cambridge, England,.19-25 September 1976, pp. 301-306. Learned In-. formation (Europe) Ltd, Oxford.

Goodwin, C. S., and Smith, B. C. (1976). Computer ${ }^{\omega}$ printing and filing of microbiology reports. I. Descrip-은 tion of the system. Journal of Clinical Pathology, 29, 543-552.

Harvey, K. J., Were, M., Heys, W., and Smith, D. D. (1972). A time-shared computer system for data $\overrightarrow{0}$ processing in bacteriology. Medical Journal of $\infty$ Australia, 2, 1076-1080.

Kunz, L. J., Poitras, J. W., Kissling, J., Mercier, B. A., Cameron, M., Lazarus, C., Moellering, R. C., and Barnett, G. O. (1975). The role of the computer in microbiology. In Modern Methods in Medical Microbiology: Systems and Trends, edited by J. E. Prier, $\mathbb{D}$ J. Bartola, and H. Friedman, pp. 181-193. University Park Press, Baltimore.

Spraberry, M. N. (1969). Computer processing of microbiology data-part of total laboratory system. American Journal of Medical Technology, 35, 77-92.

Vermeulen, G. D., Gerster, J. W., Young, V. M., and Hsieh, R. K. C. (1974). A computerized data storage? and retrieval system for clinical microbiology. $\frac{5}{3}$ American Journal of Clinical Pathology, 61, 209-215.

Whitby, J. L., and Blair, J. N. (1970). A computer-linked $\delta$ data processing system for routine hospital bacterio- ${ }_{2}$ logy. In Automation, Mechanization and Data Handling 오 in Microbiology, edited by A. Baillie and R. J. Gilbert, pp. 23-32. Academic Press, London.

Wootton, I. D. P. (1965). Computer-assisted preparation of laboratory reports. In Progress in Medical Com- $N$ puting (Symposium, Elliott Medical Automation Ltd), $N$ pp. 57-60. Blackwell, Oxford.

Requests for reprints to: Professor D. A. Mitchison, Department of Bacteriology, Royal Postgraduate Medical School, Hammersmith Hospital, London W12. 